\title{
Vínculo de la persona con cáncer- cuidador familiar (diada) durante la hospitalización: teoría fundamentada
}

\section{Vond in person with cancer - family caregiver (dyad) during hospitalization: grounded theory}

\author{
Ángela María Combita-Zambrano (iD) ${ }^{1}$, Lorena Chaparro-Díaz (iD) 2
}

\begin{abstract}
1. Instituto Nacional de Cancerología. Bogotá, Colombia. Correo: angelitacombita@gmail.com - https://orcid.org/0000-0002-0043-1316
2. Universidad Nacional de Colombia. Bogotá, Colombia. Correo: olchaparrod@unal.edu.co - http://orcid.org/0000-0001-8241-8694

Tipología: Artículo de investigación científica y tecnológica

Para citar este artículo: Combita-Zambrano AM, Chaparro-Díaz L. Vínculo de la persona con cáncer- cuidador familiar (diada) durante la hospitalización: teoría fundamentada. Duazary. 2021 enero; 18(1): 45-56. Doi: https://doi.org/10.21676/2389783X.3830

Recibido en marzo 28 de 2020

Aceptado en noviembre 11 de 2020

Publicado en línea en enero 07 de 2021
\end{abstract}

\section{RESUMEN}

Palabras

clave:

cuidadores; atención al paciente; enfermedad crónica; hospitalización; neoplasias. teoría fundamentada, investigación cualitativa.

Keywords: Caregivers; Patient Care; Chronic Illness; Hospitalizatio n; Neoplasms, Grounded .Theory, Qualitative Research.
Este se trata de un estudio que busca describir el vínculo de la diada (persona con cáncer-cuidador familiar) durante la hospitalización en un Hospital Universitario en Bogotá. Es un estudio cualitativo con enfoque de teoría fundamentada que parte de 41 entrevistas semiestructuradas a 14 personas con cáncer, 13 cuidadores familiares y 13 en pareja. Se codificaron los datos derivados de las tres fuentes de entrevistas. Luego se agruparon en subcategorías por cada fuente y al final se identificaron siete patrones que describen la forma como se da el vínculo durante la hospitalización. Se identificó que las diadas en estos momentos de crisis resignifican su relación, los afianza como equipo y les permite reconocer la necesidad de apoyo social e institucional. Este estudio contribuye a la práctica de enfermería al determinar los aspectos significativos de la diada durante la hospitalización, como disminuir las barreras del sistema, mejorar la comunicación, promover la convivencia de forma diferente, facilitar el apoyo institucional y permitir proyectarse como diada y como personas para nuevos momentos de la trayectoria.

\section{ABSTRACT}

This study aims to describe the bond of the dyad (Person with cancer - Family Caregiver) during hospitalization at Hospital Universitario in Bogotá. This is a qualitative study with a Grounded Theory approach based on 41 semi-structured interviews with 14 people with cancer, 13 family caregivers and 13 couples. The data derived from the three sources were coded. Then, they were grouped into subcategories by each source, and at the end, seven patterns were identified that describe how the bond occurs during hospitalization. It was identified that the dyads in these crisis moments give a new meaning to their relationship, strengthen them as a team and allow them to recognize the need for social and institutional support. This study contributes to the practice of nursing by determining the significant aspects of the dyad during hospitalization, such as reducing system barriers, improving communication, promoting coexistence differently, facilitating institutional support and allowing to project as a dyad and as people for new moments of the trajectory. 


\section{INTRODUCCIÓN}

El cáncer por lo general se asocia al riesgo de muerte inminente, mutilación y tratamientos agresivos. Esta enfermedad provoca una serie de debilidades en la vida de la persona que la padece, lo que implica una condición de dependencia y necesidad de que alguien le acompañe en todo el proceso de diagnóstico, convivencia y tratamiento. Ese alguien es el cuidador, quien, por lo general, es una persona de la propia casa, la más cercana, con quien el enfermo tiene mayor confianza, con quien se crea una relación de afecto, compasión y amor ${ }^{1}$.

Los cuidadores de personas con cáncer se descompensan en la medida en que se esfuerzan por cuidar, tomar decisiones y hacer tareas del hogar; además, toman responsabilidades financieras y tratan de satisfacer sus propias necesidades y mantener una rutina familiar normal ${ }^{2}$. Los cuidadores familiares, en general, son sujetos con diversos problemas de salud $y$, en muchos casos, sin ayuda y orientación. Estos problemas pueden ser una consecuencia de la falta de preparación de su nuevo rol, lo que genera estrés, síntomas depresivos, baja autoestima y autoeficacia, además de problemas de su salud física ${ }^{3}$.

De otra parte, el vínculo cuidador familiar-persona con Enfermedad Crónica No Transmisible (ECNT) no solo permanece, sino que además cambia durante su trayectoria. Se cree que en la hospitalización el rol del cuidador se transforma y se integran cuidadores formales. Ese cambio suele producir sentimientos combinados de alivio y de culpa en los familiares por querer cumplir gustos del paciente que propician la sobreprotección y, por lo tanto, el conflicto con los cuidadores formales ${ }^{4}$.

Abordar esa diada es parte de un concepto relativamente reciente en el contexto colombiano. En situaciones de cáncer surge la necesidad de reconocerla como un equipo interdependiente, en donde las dos experiencias se combinan para desarrollar comportamientos ante el manejo de la condición crónica. Esto se ha reconocido como salud diádica ${ }^{5}$ e implica que cuando se aborda al cuidador, se está reconociendo de antemano a quien cuida, es decir, a la persona en condición de cronicidad: son en sí un sujeto de cuidado. Anteriormente se han abordado cuidadores en escenarios comunitarios; sin embargo, los cuidadores de personas hospitalizadas pueden vivir experiencias muy particulares, lo que llevó a concebir la necesidad de reconocer esta experiencia en un momento de trayectoria de la cronicidad como lo es la hospitalización. Se planteó como objetivo describir el vínculo de la diada (persona con cáncer-cuidador familiar) durante el momento de hospitalización en un Hospital Universitario en la ciudad de Bogotá.

\section{MATERIALES Y MÉTODOS}

\section{Tipo de investigación}

Se optó por un estudio cualitativo con enfoque de teoría fundamentada ${ }^{6}$, porque el contexto de un escenario hospitalario está mediado por interacciones que afectan el desarrollo del vínculo. En otras palabras, se crea un fenómeno social mediado por significados que se dan en la interacción entre la diada y de esta con los cuidadores formales, otros familiares, la institución y el sistema de salud.

\section{Participantes}

Las informantes fueron 14 diadas, conformadas por 14 Personas con Cáncer (PC), hospitalizadas en los servicios de hematología, oncología, medicina interna y quirúrgica de un Hospital Universitario en Bogotá, con los siguientes criterios de inclusión: al menos 10 días hospitalizado, mayor de 18 años, que requería un cuidador familiar de manera parcial o total, sin déficit cognitivo o enfermedad mental; y 13 Cuidadores Familiares (CF) con los siguientes criterios de inclusión: mayor de 18 años, con vínculo de parentesco o cercanía con la persona con enfermedad crónica y que hubiese asumido el cuidado de la persona durante el tiempo de hospitalización. Una de las cuidadoras de un paciente no pudo participar en la entrevista semiestructurada por proceso administrativo de remisión; sin embargo, las reflexiones derivadas se integraron a los resultados. 


\section{Técnica de recolección}

La recolección de los datos se realizó entre marzo y junio de 2018 en un Hospital Universitario ubicado en Bogotá D.C, Colombia.

Se realizaron entrevistas semiestructuradas, primero a cada miembro de la diada por separado y luego una entrevista juntos. En todos los casos se estableció una conversación de tal manera que expresaran sentimientos, emociones y opiniones acerca de su relación paciente-cuidador durante el tiempo de hospitalización. Se tuvo un primer contacto con la diada y en él se explicó el objetivo del proyecto. Cuando hubo aceptación de participar, se acordaron posibles fechas de encuentro con cada uno, enfatizando en que fueran momentos sin procedimientos de rutina o especiales durante la estancia hospitalaria. Al momento del encuentro con cada uno o con la diada se confirmó el interés en participar de manera voluntaria. Entonces, se procedió a solicitar el consentimiento informado verbal y escrito, resolviendo inquietudes y solicitando autorización para la grabación. Se realizaron preguntas iniciales que fueron las mismas para los miembros de la diada: ¿Cuénteme cómo ha sido la relación con su paciente/familiar durante la hospitalización? ¿Cómo cree que se ha afectado su relación al estar hospitalizado? y ¿Cómo cree que le afecta la hospitalización en su vida diaria? A medida que se iba avanzando en la entrevista, se hicieron preguntas específicas para ampliar sobre lo que iban conversando.

En la entrevista se estuvo alerta a la comunicación verbal y no verbal del participante, permitiendo un espacio de confianza con la expresión de confidencias, dudas e inquietudes. Las entrevistas tuvieron una duración mínima de 15 minutos y máxima de 1 hora 17 minutos, para un total de 242 minutos en PC, 192 minutos en CF y 402 en diadas. El manejo de los datos inició con la transcripción de las entrevistas y la organización de las notas de campo entre 24 y 72 horas posteriores a las entrevistas.

De acuerdo con la metodología, se recurrió a un muestreo teórico en el que se fueron saturando las categorías que iban surgiendo con cada entrevista, haciendo preguntas secundarias que permitieran profundizar ${ }^{7}$. Cuando se fueron encontrando códigos repetidos dentro de las categorías, se hicieron dos entrevistas adicionales para confirmar que no existieran datos nuevos.

Se tuvieron en cuenta criterios rigor $^{8}$ de la investigación cualitativa con estrategias como la validación de cada código, la validación durante el proceso de socialización de avances con profesores del programa de Maestría en Enfermería, miembros del grupo de investigación al que pertenecen las autoras, el contraste con literatura científica cuando se iban encontrando los elementos conceptuales de cada categoría (credibilidad), la descripción detallada del contexto con la caracterización sociodemográfica, la percepción de carga y los medios de información, y la comunicación a través de la Ficha de caracterización de la diada ${ }^{9}$ que dio permiso para su utilización (transferibilidad). Además, se tuvo un registro grabado de cada entrevista y fueron trascritas textualmente. Al ser la primera experiencia de investigación cualitativa del primer autor, se acogió una propuesta metodológica de análisis sencilla y pertinente para investigadores novatos (Fiabilidad).

\section{Análisis de la información}

Antes de iniciar el análisis se realizó lectura de las entrevistas, transcripción y limpieza de estas, e identificación de fenómenos en la lectura minuciosa, línea por línea, que pudiera describir la relación de la persona con cáncer con su cuidador familiar en el contexto hospitalario. El análisis tuvo en cuenta la metodología propuesta por Strauss y Corbin $^{6}$ mediante codificación abierta (códigos nominales y su refinamiento), axial (subcategorías) y selectiva (categorías) a cada entrevista (PC, CF y Diada). Se utilizó también comparación constante y pensamiento teórico en el que fue clave considerar los seis elementos analíticos propuestos por Strauss y Corbin $^{6}$ : condiciones causales, fenómeno, contexto, condiciones intervinientes, estrategias y consecuencias. Se manejaron los datos en Atlas ti versión 7.5.4, licenciada para la Universidad Nacional de Colombia.

\section{Declaración sobre aspectos éticos}


Se contó con Aval del Comité de Ética de la Facultad de Enfermería de la Universidad Nacional de Colombia (Aval 050-17) y con el Aval institucional del Hospital Universitario. Las entrevistas fueron grabadas previo consentimiento de los participantes. El abordaje de un paciente oncológico conlleva implicaciones éticas y filosóficas; desde el punto de vista ético, el estudio tuvo en cuenta lo dispuesto en la Ley 911 de 2004, artículo 29. Se asumió además la Resolución 008430 de 1993 en donde se previó un riesgo posible de alteración emocional. Sin embargo, se previó con disponibilidad de remisión para acompañamiento psicológico que no fue requerido. En una entrevista con una PC se presentó dolor y se suspendió la sesión; no fue reprogramada. Los datos fueron manejados con confidencialidad, asignando números a los códigos y entrevistas, a los que únicamente tuvieron acceso los autores.

\section{RESULTADOS}

\section{Caracterización de la diada}

La Tabla 1 presenta el perfil de la diada en el que se encuentra que las cuidadoras mujeres en edad productiva, casadas (generalmente esposas de la $\mathrm{PC})$, de clase media, con formación primaria o secundaria, no eran las únicas cuidadoras y la mayoría no tenían experiencias previas de cuidado. Las personas con cáncer fueron generalmente hombres, adultos mayores, casados, con formación hasta profesional, con dedicación al hogar, clase media, con menos de dos años de diagnóstico y con menos de 30 días de hospitalización.

\section{Variable central y patrones identificados}

Las categorías se agruparon en las tres fuentes de información (PC, CF, diada). De la PC se encontraron 37 códigos nominales, agrupados en siete subcategorías; de los CF se derivaron 21 códigos nominales agrupados en seis subcategorías; y de las diadas se derivaron 25 códigos nominales que se agruparon en siete subcategorías (Tabla 2).

Al tener las 20 subcategorías, se seleccionaron siete que corresponden a los patrones que describe el proceso y que explican la categoría central:
"Reconociendo el valor de la hospitalización de la diada como parte del cáncer en la vida misma". Para definirlos se ubicaron en una matriz que comparaba los elementos comunes entre las categorías. Esto dilucidó que había unas categorías que eran los hitos de experiencia, independiente de la fuente (PC, CF o diada). Se definía hito por ser un momento significativo de la experiencia visible en códigos descriptores comunes (basado en los datos), que visibilizaban las interacciones entre la diada, con familia extensa y profesionales (interacción simbólica), y reflejaban aspectos nuevos de la experiencia o fenómenos ya descritos en la literatura (validez de los datos).

En el primer patrón "Afrontando las barreras del sistema", la diada se ve impresionada por los procesos administrativos y las dificultades del sistema de salud como trámites rutinarios que permiten tener oportunidad en la atención, por lo cual hace memoria de experiencias previas propias o de otra persona, e incluso en otros hospitales. Ella se acoge a las normas institucionales que le pueden servir o no en la asistencia médica, de la manera que se quiere o se necesita, ya que las dificultades en la atención no solo se presentan al ingreso o al inicio de la hospitalización, sino también en el transcurso de la misma a la par del tratamiento.

"...cuidado en ir y buscar con las EPS, es ir a hablar con el que tenga que hablar, para que me asignen aquí, para que me lo den rápido". (P.2.JH.48.126)

"... porque como hay tantos pacientes, tantas cosas por hacer entonces a veces no se toma la prioridad para ciertas personas y pues igual uno lucha por lo de uno, no...me imagino que habrán personas ahí también presionando, entonces así mismo uno debe presionar para lo de uno". (P.13.JB.05.05).

El segundo patrón "Comprendiendo la enfermedad y el tratamiento" se da en los momentos de mayor percepción de los signos y síntomas, la confirmación del diagnóstico de cáncer, el tratamiento con los exámenes respectivos y los procedimientos 0 complicaciones. Esta situación confirma la complejidad de la condición y favorece la desconfianza en el tratamiento; sin embargo, 
median aquí habilidades y lecciones de vida frente a

otros procesos de enfermedad en la PC y el CF.

Tabla 1. Caracterización de las diadas (PC-CF).

\begin{tabular}{|c|c|c|c|}
\hline \multicolumn{2}{|r|}{ Variable } & $\begin{array}{c}\text { Persona con cáncer } \\
\qquad(n=14)\end{array}$ & $\begin{array}{l}\text { Cuidador familiar } \\
\qquad(n=14)\end{array}$ \\
\hline \multirow{13}{*}{ Tipo de cáncer } & Linfoma No Hodking & 1 & \multirow[t]{13}{*}{ No aplica } \\
\hline & Linfoma B Difuso & 1 & \\
\hline & Macroadenoma Hipofisiario & 1 & \\
\hline & Carcinoma Metastásico & 1 & \\
\hline & Adenocarcinoma Biliar & 1 & \\
\hline & Tumor De Gist & 1 & \\
\hline & Cáncer Próstata & 1 & \\
\hline & Lesión Tumor Pancreático & 1 & \\
\hline & Cáncer de Mama & 1 & \\
\hline & Cáncer Gástrico Metastásico & 1 & \\
\hline & Cáncer de Colon & 2 & \\
\hline & Cáncer de Tiroides & 1 & \\
\hline & Cáncer de Recto & 1 & \\
\hline \multirow{5}{*}{ Morbilidad del cuidador } & Enfermedad respiratoria crónica y Asma & \multirow[t]{5}{*}{ No aplica } & 1 \\
\hline & Fibromialgia & & 2 \\
\hline & Hipertensión arterial & & 3 \\
\hline & Diabetes mellitus & & 1 \\
\hline & Sin enfermedad crónica & & 7 \\
\hline \multirow{2}{*}{ Género } & Femenino & 6 & 11 \\
\hline & Masculino & 8 & 3 \\
\hline \multirow{5}{*}{ Estado civil } & Soltero & 1 & 3 \\
\hline & Casado & 9 & 8 \\
\hline & Unión Libre & 2 & 2 \\
\hline & Separado & 1 & 1 \\
\hline & Viuda & 1 & 0 \\
\hline \multirow{4}{*}{ Nivel de escolaridad } & Primaria & 4 & 8 \\
\hline & Bachiller & 4 & 6 \\
\hline & Técnico & 1 & 0 \\
\hline & Profesional & 5 & 0 \\
\hline \multirow{3}{*}{ Ocupación } & Empleado & 3 & 1 \\
\hline & Hogar & 8 & 8 \\
\hline & Independiente & 3 & 5 \\
\hline \multirow{3}{*}{ Estrato socioeconómico } & 2 & 4 & 6 \\
\hline & 3 & 8 & 7 \\
\hline & 4 & 2 & 1 \\
\hline \multirow{4}{*}{$\begin{array}{l}\text { Relación del cuidador } \\
\text { con la persona con } \\
\text { cáncer }\end{array}$} & Esposa & \multirow[t]{4}{*}{ No aplica } & 8 \\
\hline & Sobrina & & 2 \\
\hline & Hija & & 3 \\
\hline & Mamá & & 1 \\
\hline \multirow{2}{*}{ Único cuidador } & Sí & \multirow[t]{2}{*}{ No aplica } & 5 \\
\hline & No & & 8 \\
\hline \multirow{3}{*}{ Horas de cuidado } & $8-10$ horas & \multirow[t]{3}{*}{ No aplica } & 3 \\
\hline & 12 horas & & 3 \\
\hline & 24 horas & & 8 \\
\hline Experiencia previa & Sí & No aplica & 6 \\
\hline
\end{tabular}




\begin{tabular}{|c|c|c|c|}
\hline & No & & 8 \\
\hline \multirow{3}{*}{ Tiempo de diagnóstico } & 1-7 meses & 8 & \multirow[b]{3}{*}{ No aplica } \\
\hline & 1-2 años & 4 & \\
\hline & $5-12$ años & 2 & \\
\hline \multirow{3}{*}{$\begin{array}{l}\text { Tiempo de } \\
\text { hospitalización }\end{array}$} & 8-10 días & 5 & \multirow[b]{3}{*}{ No aplica } \\
\hline & 15 -30 días & 7 & \\
\hline & 90 días & 2 & \\
\hline \multirow[t]{4}{*}{ Edad (años) } & $30-40$ & 3 & 3 \\
\hline & $41-60$ & 2 & 7 \\
\hline & $61-80$ & 8 & 4 \\
\hline & +81 & 1 & 0 \\
\hline
\end{tabular}

Tabla 2. Subcategorías derivadas según fuente de datos

\begin{tabular}{|c|c|c|}
\hline Subcategoría de PC & Subcategoría del CF & Subcategoría de la diada \\
\hline Afrontando las barreras del sistema & $\begin{array}{l}\text { Considerando una mirada } \\
\text { retrospectiva }\end{array}$ & Volviendo la mirada atrás \\
\hline $\begin{array}{l}\text { Sintiendo diferentes expectativas del } \\
\text { tratamiento }\end{array}$ & $\begin{array}{l}\text { Comprendiendo la enfermedad y el } \\
\text { tratamiento }\end{array}$ & $\begin{array}{l}\begin{array}{l}\text { Descifrando el recorrido de la } \\
\text { enfermedad }\end{array} \\
\end{array}$ \\
\hline Cambiando mi vida y ser & $\begin{array}{l}\text { Reorganizando la relación con el } \\
\text { paciente }\end{array}$ & Conviviendo en otro espacio \\
\hline Pensando en la familia & Reconstruyendo una nueva rutina & Replanteando el vínculo de cuidado \\
\hline Reconociendo al cuidador & Requiriendo apoyo de otros & Fortaleciendo la red de apoyo \\
\hline Viviendo entre el personal de salud & Proyectándose al futuro & Refiriendo un soporte intangible \\
\hline Aprendiendo del proceso & & $\begin{array}{l}\text { Adquiriendo habilidades para } \\
\text { continuar }\end{array}$ \\
\hline
\end{tabular}

“...al principio como te dije, me dio bastante duro, por lo que pues, me detectaron, pero pues el apoyo de mi familia fue esencial y todo, y pues ya me calmé y ya no le puse como tanto color a pensar en eso y salir adelante, entonces pues, ya me tranquilicé más y pues ahí voy, en recuperación y pues ya, gracias a Dios estoy bastante mejor". (P.6. JhC.14.04).

"Entonces, me asusta la infección, me asusta a tal punto de que he tratado de comunicarme para ver si yo mismo puedo comprar los medicamentos y que sean aplicados, para mantenerme en esa calidad de vida, en esa seguridad, en la seguridad de que no me va a afectar para poder continuar con el tema tratamiento, con la propia vida". (P.2.JH.33.88).
En el tercer patrón "Conviviendo en otro espacio" se experimentan cambios de roles en la medida en que se adaptan día a día al nuevo ambiente, ya que la dinámica de convivencia cambia de la casa al hospital. Ahora se piensa en cómo estará el hogar y cada uno de sus integrantes que le acompañan desde la distancia. En este nuevo espacio se comparte con otras personas como son familiares que no veían con frecuencia, otros pacientes con sus familiares y cuidadores formales. Esta convivencia se da entre procedimientos, dispositivos biomédicos, habitaciones especiales de aislamiento, espacios de alimentación y de actividades de la vida diaria que van cambiando según el tiempo de permanencia en el hospital. 
"Pues para nosotros también fue un poquito impactante porque pues tampoco nos habíamos visto abocados a esto (a la hospitalización) y la primera vez que lo mandaron allá fue acompañado y la gente que estaba al lado era una gente muy bullosa, muy escandalosa y nunca habíamos estado en esa experiencia, entonces nos alteramos bastante también, es más tranquilo solos nosotros dos, es más tranquilo". (D.12.JMML.21.08).

En la medida en que se permanece en el hospital la diada sigue el cuarto patrón "Replanteando el vínculo de cuidado", que se hace evidente a medida que se perciben mutuamente cambios del paciente y del cuidador: el paciente con su padecimiento de la enfermedad y el familiar adoptando su rol de cuidador. Esto se expresa manteniendo supervisión, compensando la carga y entendiendo al otro. La diada reconoce una nueva forma de amor y en ocasiones se retoma la confianza, se resaltan las virtudes y hay agradecimiento al cuidador, que de manera especial compensan la sobrecarga existente.

"entonces ahí estamos viendo quién viene en la mañana, las cosas, la ropa, estar pendiente de hablar con los médicos, qué medicamentos, qué le van a hacer, que ya... El viernes pasado me presentó una convulsión, entonces me pegó mucho susto y yo dije -no, él no se puede quedar solo esta noche-, porque no había quien se quedará en la noche, pero ese día le tocó al hijo de él quedarse porque yo lo vi un poquito malito..." (D.5.ABEB.38.21).

"Sí, eso cansa mucho, y digamos uno se desgasta mucho, uno de acompañante es desgastante sea como sea es desgastante..." (D.11.MDGM.64.30).

"Yo ya le expliqué a sumercé, ese dolor que tengo por verlo así, por lo que tienen que hacer y por lo que tiene, ese dolor en mí despierta más amor por él, ser más especial con él" (D.3.RSAA.50.38)

Para seguir enfrentando como diada entran al quinto patrón "Requiriendo apoyo de otros", que es un llamado que hace la diada y especialmente el cuidador principal a los cuidadores familiares $y$ formales. Se hace visible como requerimiento porque la familia percibe cansancio; el cuidador reclama por ayuda institucional en procedimientos y "estar pendiente". La forma en que el cuidador compensa este requerimiento es acudiendo a su responsabilidad y compromiso con la PC de servir a él y a Dios.

"pero están pendientes de él, cada rato me llaman, que pobrecita sumercé allá, ay Dios mío, que se nos va a enfermar, yo les digo, que, eso no pasa nada. (Risas)..." (C.7.CV.25.19)

"Yo que día, discutía con un auxiliar porque yo salí de la habitación y le dije - yo me voy, te recomiendo que mi mamá queda sola-, es que a veces ella timbra $y$ son quince a veinte minutos". (C.1.DG.23.64).

"Entonces, para mí no es una carga mi esposa, la amo y me parece, es una enseñanza cristiana del Señor, el Señor también nos enseña que es mejor servir a otros que, lo sirvan a uno, si". (C.13.GE.50.148)

Desde el diagnóstico del cáncer y todo el proceso de hospitalización, la diada experimenta el sexto patrón "Refiriendo un soporte intangible", relacionado con emociones, pensamientos, sentimientos y sostén espiritual, en los que se padecen altibajos, se media entre la limitación y el espacio propio, y se equilibra el dolor con el crecimiento personal. Se aprecia cierta madurez familiar por la situación de ausencia y de enfermedad. Al comunicarse las diferentes dificultades durante los espacios de visita, interpretando la información y acompañando en las aflicciones, la familia valora el rol del cuidador y permite superar sentimientos negativos con soporte espiritual. Aquí los sujetos más frágiles de la familia (los niños y las mascotas) ayudan a resignificar las emociones y dan sentido a la vida.

"Soy hijo adoptado, ella es tía, mi mamá es hermana y mi mamá biológica falleció hace tres años y nuestro núcleo familiar éramos los tres. Entonces, ya tuvimos esa pérdida hace tres años y sí, pues somos unidos, y nos une la fe en Dios sobre todo, somos muy creyentes, entonces eso es lo que nos da la paz y la fortaleza para cualquier prueba que nos pone la vida". (D.8.AOFO.21.04). 
Para poder darle sentido a la vida la diada llega a un séptimo patrón: "Adquiriendo habilidades para continuar". Esto se da, inicialmente, aceptándose a sí mismo y confiando en los demás (familiares / Institución), como preparación para el egreso e incluso un posible reingreso debido al ciclo de los tratamientos. Sin embargo, al ser conscientes de esta realidad, a partir de la hospitalización se emprende un nuevo camino basado en la reflexión, reconociendo ganancias del proceso, fortaleciendo la relación, cuidando de su salud con el ánimo de ser ejemplo para otros y reconociendo que, más allá de un lugar o de una situación de enfermedad, hay un futuro con un proyecto de vida propuesto.

"cuando en realidad uno no sabe en qué momento cambian las cosas, y esas cosas vienen a mi mente y yo he recapacitado mucho, yo digo: Dios mío, de verdad a veces uno es muy necio, o a veces uno, yo por ejemplo soy de las personas que son muy arraigadas al trabajo, yo soy muy responsable, yo trabajo y yo creo que eso también me afectó mucho porque yo empecé a trabajar y ese trabajo me absorbió bastante". (D.13.JBGR.17.21).

"Entonces le enseña a uno a ser más tolerante, más paciente, y eso a ser una mejor persona. Esto definitivamente lo fortalece mucho a uno, se vuelve uno mejor persona en todo sentido". (D.1.YHDG.44.72).

\section{DISCUSIÓN}

Los resultados de este estudio confirman aspectos generales del perfil sociodemográfico en el que se encuentra la feminización del rol de cuidador: las esposas asumen el rol de cuidadoras de personas que son de más edad que ellas ${ }^{10}$. En Colombia se han encontrado situaciones de vulnerabilidad de los cuidadores, en cuanto a la pobreza y nivel socioeconómico, que en este estudio no se apreciaron. Sin embargo, sí se aprecia una situación de vulnerabilidad de mujeres cuidadoras que es el bajo nivel de escolaridad ${ }^{11}$.

En este escenario, la diada atraviesa por diferentes etapas determinadas por la complejidad de la enfermedad y otras que tiene que afrontar por las barreras del sistema, como procesos administrativos y/o situaciones del sistema de salud, antes, durante y al egreso de la hospitalización. Según cifras del Instituto Nacional de Cancerología (INC) en el 2015, el 6,3\% de los hogares colombianos tiene barreras de acceso a los servicios de salud y este porcentaje varía por departamento ${ }^{12}$. Este estudio fue realizado en una institución con usuarios afiliados a una aseguradora de régimen contributivo de estrato socioeconómico 3 y 4 , por lo que los resultados pueden distar un poco, aunque se mantienen barreras administrativas.

La identificación de signos y síntomas, que en esta investigación fue reconocida por la diada, dista un poco de poblaciones vulnerables en las que el conocimiento acerca de factores de riesgo y los síntomas del cáncer son mucho más bajos ${ }^{12}$.

Los cambios biopsicosociales influyen en la desconfianza de las intervenciones. Es así que contar con una etiqueta diagnóstica representa paradójicamente una gran satisfacción y bienestar, porque les permite familiarizarse con diferentes términos acerca del cáncer como de su tratamiento, y ayuda a definir un patrón de respuesta que puede incluir una fase final de vida ${ }^{13}$. Nuestros resultados muestran que los primeros meses de la experiencia son el momento propicio para intervenciones de tipo psicoeducativo, que deben ser constantes y no limitadas al egreso hospitalario. Suess et $a l^{14}$ confirman que en situaciones de cáncer las fases más críticas para la insatisfacción fueron la de detección y diagnóstico, lo que ratifica que estos momentos deben ser mejor acompañados por los profesionales de la salud, pues en ocasiones se deja a la diada demasiado tiempo en incertidumbre y esto afecta su experiencia.

Al convivir en otro espacio se presenta cambio de roles y una adaptación día a día al nuevo ambiente: la dinámica de convivencia cambia de la casa al hospital. Esta investigación se enfocó en un cuidador principal; no tuvo en cuenta otros cuidadores que hacen parte de la experiencia, como son los cuidadores a distancia que se ocupan de quehaceres del hogar, soporte económico o transporte ${ }^{15}$. Los resultados también mostraron que el cuidador hace un requerimiento de apoyo a otros, es decir, hace un 
llamado a mayor apoyo social de la familia extensa y de los cuidadores formales. Estos hallazgos indican que es necesario contar con políticas institucionales para el reconocimiento de las necesidades de los cuidadores, que siguen siendo invisibilizados y poco valorados.

En esta investigación el cuidado instrumental dentro del hospital es el motor de la relación tanto entre la diada, como con otros cuidadores familiares y formales. Esto genera percepción de carga y el cuidador hace un llamado para que se dé más empatía por parte del paciente y del cuidador profesional. En el cáncer, los estudios se enfocan más en el cuidado paliativo, con el que se han explorado tipos de relaciones empáticas entre personal de salud y cuidadores. Una de estas exploraciones es el estudio de Wittenberg-Lyles et $a l^{16}$ en el que se resalta la necesidad de transcender el comunicar los aspectos que mejoran la calidad de vida del paciente hacia asesoramiento terapéutico e intervenciones psicoeducativas dirigidas al cuidador. Al ser la hospitalización una situación de corto plazo, puede que no se tenga interés en establecer relaciones medidas por sentimientos.

Cuando una diada convive en el hospital día y noche puede verse afectada en su salud psicosocial, relacional y física. Asumir este rol en el hospital implica una transición que puede ser una oportunidad para entenderse y agradecerse en medio de la adversidad. Esto implica adquirir autocontrol, el cual ha sido descrito por Bidwell et $a 1^{17}$ y que podría ser un elemento clave para las intervenciones con diadas, como asumir la autogestión de la salud con autocontrol.

Este estudio se ubicó en un escenario poco común para abordar a los cuidadores: el hospital. No se identificaron estudios que dieran cuenta del patrón "Conviviendo en otro espacio", lo que es un aporte para entender que la trayectoria integra el contexto y la convivencia con otras personas significativas que se vuelven los cuidadores formales e inclusive los que solo preguntan a la distancia por el paciente. Se ha documentado que el soporte social es un factor relevante en la experiencia de la diada. Se afirma que cuando el paciente tiene mejor soporte social, la salud del cuidador mejora, y cuando el cuidador tiene apoyo social, los pacientes reportan mejor salud autoinformada ${ }^{17}$. De otra parte, los profesionales de enfermería fueron fuente de información y orientación: en la investigación se centraron en el apoyo para el cuidado físico. Sin embargo, Kelley et $a l^{18}$ resaltan la necesidad de dar soporte emocional a los cuidadores, pues permitirá una estabilidad del paciente y por lo tanto mejores relaciones. Los resultados de este estudio confirman la necesidad de continuar con estrategias de apoyo social tangible, al igual que el intangible que en este estudio fue valioso y permitió sostenerse en el cuidado.

Cuando se piensa en las habilidades para continuar, estas se adquieren desde antes de un diagnóstico de cáncer e incluso antes de ingresar al hospital. No obstante, se van afianzando, reforzando o aprendiendo durante el proceso de hospitalización en la preparación para el egreso e incluso para continuar el proyecto de vida, por lo cual la intervención de enfermería es importante para comprender y capacitar en acciones de cuidado que permitan fortalecer el vínculo de la diada al fortalecer la confianza en sí mismos de una manera integral, según lo que proponen Reblin et $a l^{19}$.

Un componente de interés reciente es la competencia o las habilidades de la diada. Estas habilidades están mediadas por aspectos cognitivos e instrumentales que en muchas ocasiones son asociadas a comprender la enfermedad, pero también a quien vive la condición, la autorreflexión del rol (de cuidar y de ser cuidado), los recursos disponibles y la proyección de futuro de la diada. Se ha documentado que estas habilidades implican el ámbito de la salud (como es el caso de este estudio con la hospitalización y el egreso hospitalario) ${ }^{20}$, las dimensiones domésticas asociadas a la rutina del hogar, las dimensiones organizacionales que implican la programación cuidadosa del día a día, y la dimensión emocional y el bienestar que es el lidiar con sus emociones ${ }^{21}$.

Se puede concluir que el vínculo de la diada en el escenario hospitalario es una oportunidad para consolidar la relación. Se inicia con una turbulencia de factores externos asociados al diagnóstico y al sistema de salud, vistos como barreras que, una vez se tiene un tratamiento claro y son resueltas, entienden el proceso. Aquí es clave la comunicación 
de los profesionales de la salud y especialmente el profesional de enfermería, hay momentos de riesgo de declinar y es por eso por lo que el cuidador pide apoyo de otros. El hospital definitivamente es un escenario atípico para la diada; acomodarse implica convivir y ajustar sus roles, además de recibir apoyo intangible que le da sostén y le permite proyectarse al egreso y los proyectos de vida.

Finalmente, se puede decir que estudio permitió contribuir con la propia experiencia de los participantes a comprender la forma como se desarrolla el vínculo como diada (PC-CF) y tener un momento de reflexión frente a lo que significa tener cáncer y cuidar personas con cáncer en el marco de una relación de cuidado. Este es un paradigma que el modelo biomédico no ha podido acompañar y resolver sus mayores dificultades; por lo que resulta el mayor aporte al conocimiento de la ciencia de enfermería. Por eso, tener en cuenta a la diada (PC$\mathrm{CF}$ ) como sujeto de cuidado en la institución, con base en su experiencia, permite brindar una atención de enfermería de mayor impacto y autonomía, buscando disminuir la carga de cuidado en cada uno de los integrantes de la diada, así como en su familia.

Las limitaciones del estudio es que no son generalizables sus resultados a todas las condiciones de cáncer o a instituciones hospitalarias. En este estudio se tuvieron más diadas de clase media, lo cual puede diferir de otro tipo de cuidadores en situaciones de pobreza o que vivan en ciudades que no tienen acceso a alta tecnología

\section{AGRADECIMIENTOS}

A las directivas y personal de enfermería del Hospital Universitario donde se realizó el estudio.

Al grupo de investigación Cuidado de Enfermería al Paciente Crónico de la Facultad de enfermería de la Universidad Nacional de Colombia.

A cada una de las personas con cáncer y cuidadores familiares que conformaron las diadas que participaron: por su tiempo, por su confianza y el deseo de poder contribuir a mejorar las condiciones de salud y atención de quienes tienen diagnosticado el cáncer.

\section{DECLARACIÓN SOBRE CONFLICTOS DE INTERESES}

Los autores no tienen fondos o conflictos de interés para divulgar.

\section{CONTRIBUCIÓN DE LOS AUTORES}

Primer autor: investigador principal, trabajo de campo, análisis y redacción inicial;

Segundo autor: director de tesis, análisis y redacción final.

\section{REFERENCIAS BIBLIOGRÁFICAS}

1. Valbuena C, Chaparro-Diaz L. Carga del cuidado en la díada en situaciones de cáncer. PSIC. 2018 Sep; 15(2): 361-372. Disponible en: https://revistas.ucm.es/index.php/PSIC/article/vie w/61441

2. Tiago de Oliveira W, Misue Matsuda L, Aparecida MC. Relationship between wellness and sociodemographic characteristics of caregivers of people with cancer. Invest. Educ. Enferm. 2016 Feb; 34(1): 128-136. Doi https://dx.doi.org/10.17533/udea.iee.v34n1a15

3. Ferreira NML, Dupas G, Costa DB, Sanchez KOL. Câncer e família: compreendendo os significados simbólicos. Ciênc Cuid Saúde. 2010 Sep; 9(2):26977.

Doi: https://doi.org/10.4025/cienccuidsaude.v9i2.8749

4. Chaparro L. Cómo se constituye el "vínculo especial" de cuidado entre la persona con enfermedad crónica y el cuidador familiar. Aquichán. 2011 Abr; 11(1): 7-22. Doi: http://dx.doi.org/10.5294/aqui.2011.11.1.1 
5. Lyons KS, Lee CS. The theory of dyadic illness management. J Fam Nurs. 2018 Feb; 24(1), 8-28. Doi: http://dx.doi.org/10.1177/1074840717745669

6. Strauss A, Corbin J. Bases de la investigación cualitativa. Técnicas y procedimientos para desarrollar la Teoría Fundamentada. Medellín: Universidad de Antioquia; 2002.

7. Morse JM. Asuntos críticos en los métodos de investigación cualitativa. Medellín: Universidad de Antioquia; 2003

8. Guba EG, Lincoln YS. Effective evaluation: Improving the usefulness of evaluation results through responsive and naturalistic approaches: Jossey-Bass; 1981.

9. Chaparro L, Sánchez B, Carrillo GM. Encuesta de caracterización del cuidado de la diada cuidador familiar-persona con enfermedad crónica. Rev. Cien. Cuidad. 2014 Sep; 11(2): 31-45. Doi: https://doi.org/10.22463/17949831.196

10. Chaparro L, Barrera-Ortiz L, Vargas-Rosero E, Carreño-Moreno SP. Mujeres cuidadoras familiares de personas con enfermedad crónica en Colombia. Rev. cienc. cuidad. 2016 Jun; 13(1): 72-86. Disponible en: https://revistas.ufps.edu.co/index.php/cienciaycuid ado/article/view/736

11. Carreño S, Chaparro L. Agrupaciones de cuidadores familiares en Colombia: perfil, habilidad de cuidado y sobrecarga. Pensam psicol. 2017 Ene; 15(1): 87-101. Disponible en: https://revistas.javerianacali.edu.co/index.php/pen samientopsicologico/article/view/1393

12. Instituto Nacional de Cancerología -INC. Análisis de Situación del Cáncer en Colombia 2015. Bogotá D.C., Ministerio de Salud y Protección Social. 2017. Disponible en: https://www.cancer.gov.co/Situacion_del_Cancer_ en_Colombia_2015.pdf
13. Comin L T, Panka Marina, BV, Steffani JA, Bonamigo EL. Percepción de los pacientes oncológicos sobre la terminalidad de la vida. Rev. Bioét. 2017 Aug; 25(2): 392-401. Doi: https://doi.org/10.1590/1983-80422017252199.

14. Suess A, March JC, Prieto MA, Escudero MJ, Cabeza E, Pallicer A. El proceso asistencial de cáncer: necesidades y expectativas de los usuarios. Oncología (Barc.) [Internet]. 2006 Oct; 29(9): 13-23. Disponible en: http://scielo.isciii.es/scielo.php?script=sci_arttext\& pid=S0378-48352006000900002\&lng=es.

15. Douglas SL, Mazanec $P$, Lipson A, Leuchtag $M$. Distance caregiving a family member with cancer: $A$ review of the literature on distance caregiving and recommendations for future research. World J Clin Oncol. 2016 Apr; 7(2): 214-219. Doi: https://doi.org/10.5306/wjco.v7.i2.214

16. Wittenberg-Lyles E, Debra PO, Demiris G, Rankin A, Shaunfield S, Kruse RL. Conveying empathy to hospice family caregivers: team responses to caregiver empathic communication. Patient Educ Couns. 2012 Oct; 89(1):31-7. Doi: http://dx.doi.org/10.1016/j.pec.2012.04.004

17. Bidwell JT, Lyons KS, Lee CS. Caregiver wellbeing and patient outcomes in heart failure: A metaanalysis. J Cardiovasc Nurs. 2017 Jul; 32(4): 372-382. Doi:

https://doi.org/10.1097/JCN.0000000000000350

18. Kelley $D$, Kent E, Litzelman K, Mollica MA, Rowland JH. Dyadic associations between perceived social support and cancer patient and caregiver health: An Actor-Partner Interdependence modeling approach. Psychooncology. 2019. Jul; 28(7): 14531460. Doi: https://doi.org/10.1002/pon.5096

19. Reblin M, Clayton MF,Xu J, Hulett JM, Latimer S, Donaldson G, Ellington L. Caregiver, patient, and nurse visit communication patterns in cancer home hospice. Psychooncology. 2017. Dec; 26(12) : 22852293. Doi: https://doi.org/10.1002/pon.4361 
20 Santamaría NP, García LE, Sánchez B, Carrillo G. Percepción del cuidado de enfermería dado a los pacientes con cáncer hospitalizados. Rev. latinoam. bioet, 2016. Dic; 16(1):104-127. Doi: http://dx.doi.org/10.18359/rlbi.1443

21. Meunier-Beillard N, Ponthier N, Lepage C, Gagnaire A, Gheringuelli F, Bengrine L, et al. Identification of resources and skills developed by partners of patients with advanced colon cancer: a qualitative study. Support Care Cancer. 2018. Dec; 26(12):4121-4131.

Doi:

http://dx.doi.org/10.1007/s00520-018 